\title{
Determining the Source of Dips Using Data of One Monitor in the MV Network
}

\author{
L.E. Weldemariam, V. Cuk, and J.F.G. Cobben \\ Department of Electrical Engineering \\ Eindhoven University of Technology \\ P.O. Box 513, 5600 MB, Eindhoven, The Netherlands \\ e-mail: 1.e.weldemariam@tue.nl
}

\begin{abstract}
This work discusses about a method that could be used to determine the source of voltage dips based on voltage and power measurements from a single monitor in the MV distribution network. The method uses information of activepowers of each feeder connected to the primary substation measured before, during and after the dip events. The ratios of during-dip and post-dip powers to the pre-dip power are used to distinguish the origin and cause of the disturbance. As a power quality problem related to the highest financial losses for industrial customers, knowing the origin and cause of voltage dips is very important in order to identify the weakest part(s) of the network and to take corrective measures that improve the power quality levels and reduce the potential impact of dips to customers.
\end{abstract}

Index Terms-- monitoring system, MV-network, voltage dip, voltage dip cause, voltage dip origin.

\section{INTRODUCTION}

Voltage dip is one of the power quality (PQ) disturbances which is defined as the temporary reduction in the RMS voltage below a specified threshold followed by its quick recovery [1], [2]. It can be caused by the short-duration increase in current magnitude due to the starting of heavy loads (e.g. motors), reconnection of transformers, but mainly by short-circuit faults occurring in the networks [2]. A dip originating at one part of the network can propagate to the other parts of the network and can cause the disruption of processes that consist of sensitive devices [3-6] which leads to huge financial losses [7].

The growing interest in power quality (PQ), a topic of great importance for future smart grids, has demanded the network operators to install monitoring tools at their networks in order to measure more data continuously for long periods. This helps the network operators to get ample of information about the quality of the networks in order to provide the supply with sufficient quality. In fact, voltage dips have been monitored since 2006/07 in the HV and MV of the Dutch networks, and more monitors are installed for dip measurements in the MV-networks in 2014 and 2015. For the distributed system operators (DSOs), it is crucial to know the source of voltage dips monitored at their networks- a first step in responsibility sharing to tackle the voltage dip problems affecting the end-users. Moreover, identifying the origin of dips is very important in order to identify the weakest part(s) of the network- a gateway to take corrective measures to enhance the quality of the supply and hence the performance of end-users. It is also useful in order to provide appropriate information to the customers for choosing optimal mitigation techniques to improve the PQ levels. The process of identifying the SOURCE of voltage dips includes detecting the relative location (ORIGIN) and the CAUSE of the event. The origin of the event refers to where the event originates while the cause refers to the kind of disturbance that brings about the dip event.

Monitoring dips at every point of connection (POC) could provide very accurate result of dip origin and cause. Because of economic constraint, the number of monitors installed in the MV distribution networks is restricted and this makes difficult to pinpoint the exact event location. From the data of a monitoring system in the MV distribution network, placed at the optimal location [8], voltage dip parameters including magnitude, duration and type can be obtained by diagnosing and characterizing the instantaneous values of voltage waveforms of the data [9], [10]. The data may include dips originating from the $\mathrm{HV}$ - or MV-networks which may be caused by short-circuit faults, energizing of transformers or switching of heavy loads.

To locate the source of voltage dips, researchers have proposed various techniques. In [11], a method using the real current component is discussed to locate the source of voltage dips. Another method proposed in [12] uses the line-fitting parameters of current and voltage during voltage sag for detecting sag direction. By detecting the seen impedance and its angle before the dip event, application of distance relay method is proposed in [13]. In [14], an approach based on the causes of events is considered to identify the source of voltage dips. A method based on the concurrent monitoring of an event by nearby primary substations connected to a common HV-network is discussed in [15]. However, these methods use information of multiple monitors, or require knowledge of HV-network topology or they are applied only to dips caused by short-circuit faults. 
This paper discusses the characteristics of various types of disturbances causing voltage dip problems using computer simulations and analysis of field-measured data. The method proposed to identify the source of voltage dips, using one monitor in the MV-network, is analysed. Then, the method is also applied to a four-year dataset of voltage dips monitored at the point of common coupling (PCC) of a MV distribution network to distinguish dips originating in the $\mathrm{HV}$ - and MV-networks caused by different means.

\section{NeTWORK STRUCTURE AND MONITORING}

Fig. 1 shows a simplified schematic of the electrical network modelled using DIgSILENT PowerFactory to study the characteristics of voltage dips in order to define their sources. It comprises parts of HV- and MV-networks where important parameters of the model are summarized in TABLE I. The $10 \mathrm{kV}$ feeders, operating radially, are connected to the PCC at primary substation where a monitoring system is installed. Most end-users are connected to the MV busbars through Dyn transformers.

The dip events can emanate from the HV- or MVnetworks. In this paper, the relative origin of the dip is defined as upstream when the dip originates from the HVnetwork (area within the TSO) and downstream when the it emanates from the MV-network (area within the DSO).

\section{Methodology}

As discussed in [10], [16], voltage dips caused by shortcircuit faults have typical characteristics from dips caused by motor starting and transformer excitation. This implies that it is possible to get indications about the source of voltage dips from the shape the RMS voltages. However, the proposed method is to detect the cause and origin of dips without checking the shapes of RMS voltages.

Results presented in this paper are based on computer simulations and analysis of dataset of field measurements. First, simulations are performed to verify if the proposed method works. Then, the method is applied with field-data to classify dips originating from the $\mathrm{HV}$ - and $\mathrm{MV}$ networks.

\section{A. Computer Simulations}

The proposed method uses information of active-powers of all outgoing feeders at the PCC, measured before, during and after the events, to distinguish the relative origin (HVor MV-network) and the cause of the voltage dip problems. To validate this, the electrical network in Fig. 1 is modelled and computer simulations are performed with different types of disturbances in the MV- and HVnetwork. For each disturbance, the instantaneous voltages

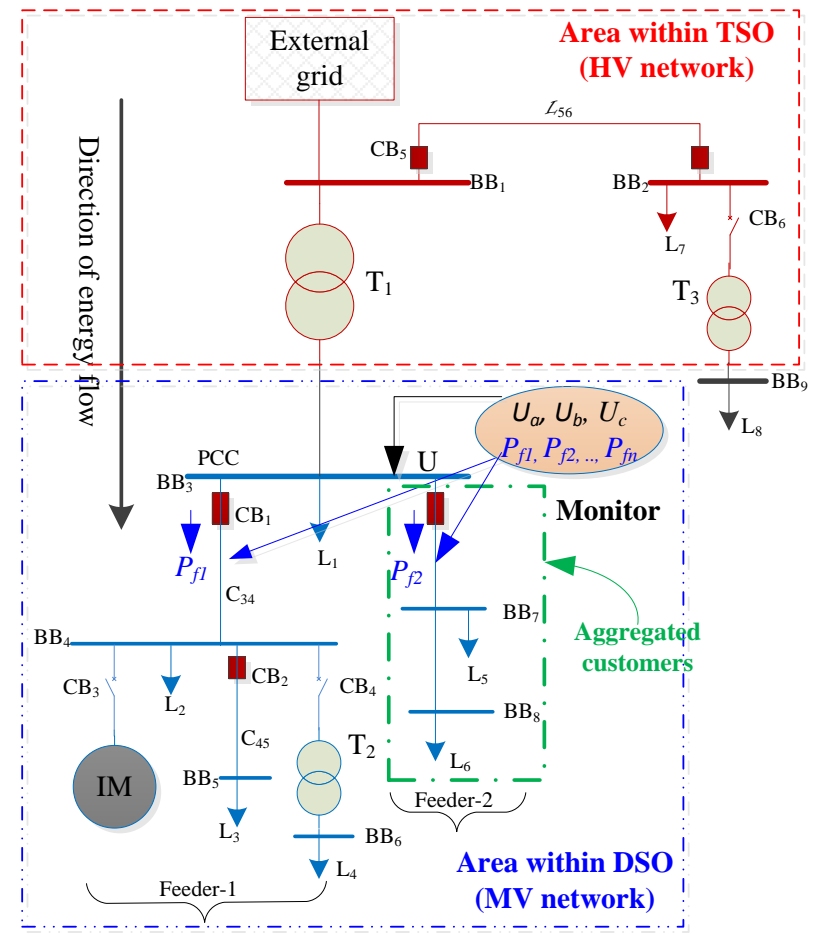

Fig. 1. Simplified representation of an electrical network for simulation

at the PCC and active powers of the feeders are measured. Information of dip-duration and shape of RMS voltages are obtained from the instantaneous voltages waveforms measured at the PCC. Likewise, activepowers of each feeder before the dip, during the dip and after the dip are obtained. In Fig. $1, P_{f 1}$ and $P_{f 2}$ are shown denoting the powers of feeder- 1 and feeder- 2 to illustrate the influence of dips emanating from feeder- $1\left(f_{1}\right)$ of the MV-network and from the HV-network.

To demonstrate voltage dips originating from the MVnetwork, with one monitor at the PCC $\left(\mathrm{BB}_{3}\right.$ of Fig. 1$)$, a balanced short-circuit fault is created at the busbar $\mathrm{BB}_{5}$ and circuit breaker $\mathrm{CB}_{2}$ is set to clear the fault within 300ms. During the dip event, power in the same feeder $\left(f_{1}\right)$ is expected to increase while power of other feeders ( $f_{2}$ in this case) is predicted to decrease. When $\mathrm{CB}_{2}$ clears the fault, it is expected feeder- 1 to lose loads, at least those represented by $\mathrm{L}_{3}$. Disturbances caused by motor starting and transformer energizing in the MV-network are studied during the reconnection of an induction motor (IM) and a transformer $\mathrm{T}_{2}$ to the busbar $\mathrm{BB}_{4}$ with switching devices $\mathrm{CB}_{3}$ and $\mathrm{CB}_{4}$. Moreover, disturbances from the HV-network caused by short-circuit faults and excitation of a transformer are considered. In this case, circuit breaker $\mathrm{CB}_{5}$ is set to react within $100 \mathrm{~ms}$ for disturbances due to short-circuit fault on the line $\left(L_{56}\right)$ connecting $\mathrm{BB}_{1}$ with $\mathrm{BB}_{2}$; and $\mathrm{CB}_{6}$ for the reconnection of transformer $\mathrm{T}_{3}$ supplying loads represented by $\mathrm{L}_{9}$. The results of simulations are analysed in the result section.

TABLE I. SUMMARY OF MODEL PARAMETERS

\begin{tabular}{|l|l|l|l|}
\hline \multicolumn{1}{|c|}{ Transformer } & Protection device & \multicolumn{1}{c|}{ Line $/$ Cable } & \multicolumn{1}{c|}{ Load } \\
\hline $\mathrm{T}_{1}: 110 / 10 \mathrm{kV}, 25 \mathrm{MVA}, \mathrm{Y}_{\mathrm{N}} \mathrm{d}$ & $\mathrm{CB}_{2}: 300 \mathrm{~ms}$ & $\mathrm{~L}_{56}=20 \mathrm{~km}$ & $\mathrm{~L}_{1}=6.5 \mathrm{MVA} ; \mathrm{L}_{2}=5 \mathrm{MVA} ; \mathrm{L}_{3}=1.5 \mathrm{MVA}$ \\
\hline $\mathrm{T}_{2}: 10 / 0.4 \mathrm{kV}, 2.0 \mathrm{MVA}, \mathrm{Dy}_{\mathrm{n}}$ & $\mathrm{CB}_{5}: 100 \mathrm{~ms}$ & $\mathrm{C}_{34}=1 \mathrm{~km}$ & $\mathrm{~L}_{4}=1.33 \mathrm{MVA} ; \mathrm{L}_{5}=4.5 \mathrm{MVA} ; \mathrm{L}_{6}=3.5 \mathrm{MVA}$ \\
\hline $\mathrm{T}_{3}: 110 / 10 \mathrm{kV}, 25 \mathrm{MVA}, \mathrm{Y}_{\mathrm{N}} \mathrm{d}$ & & $\mathrm{C}_{45}=1 \mathrm{~km}$ & $\mathrm{~L}_{7}=10 \mathrm{MVA} ; \mathrm{L}_{8}=12.2 \mathrm{MVA} ; \mathrm{IM}=1.3 \mathrm{MW}, 1500 \mathrm{rpm}$ \\
\hline
\end{tabular}




\section{B. Monitoring Data}

A PQ monitor installed at the primary substation of the MV-network (Fig. 1) records waveforms of phase-voltages sampled at $4 \mathrm{kHz}$ and half-cycle RMS values of per-phase powers of each feeder connected to the main substation. For each voltage event, the dip parameters (magnitude, duration) are obtained using the guidelines recommended by the IEC 61000-4-30 [17]. When the monitor records a dip at the PCC of the distribution network, the active power of a feeder during and after the dip event may increase or decrease (see Fig. 2) depending on the location of the disturbance and its impact to customers.

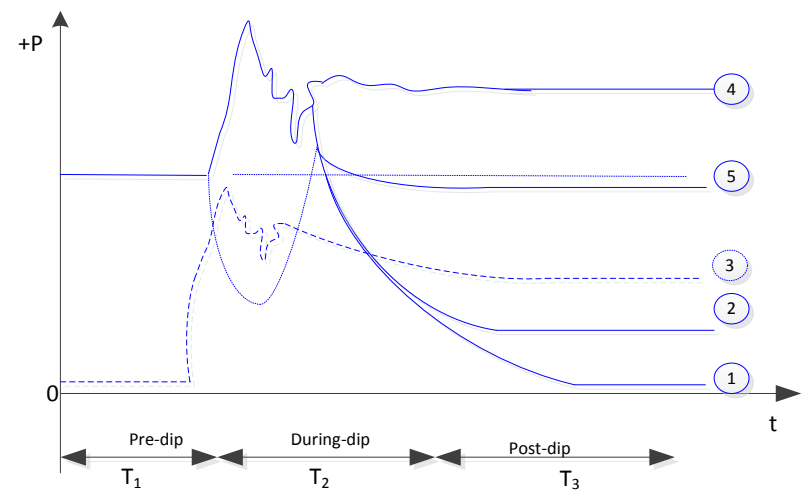

Fig. 2. Situations of a feeder during a voltage dip event

As illustrated in Fig. 2, powers of the outgoing feeders may indicate

- complete interruption of a feeder indicated by (1),

- partial loss of customers in a feeder shown by (2),

- reconnection of a new feeder as depicted by (3),

- connection of additional loads to a feeder as in (4), or

- a feeder negligibly affected by the dip, shown by (5).

The flow chart illustrated in Fig. 3 is used to distinguish the origin and cause of the disturbances using the activepower method. When applying this method, customers of each feeder are represented by one aggregated customer, and the ratios of during-dip and post-dip powers to pre-dip powers of each feeder are evaluated for each voltage dip event. The flow-chart indicates that a dip originates from the MV-network if the during-dip power of any feeder is higher than the pre-dip power; and it is caused by shortcircuit fault if the post-dip power of that feeder is lower than the pre-dip power.

\section{AnAlysis OF RESUlts}

\section{A. Simulation Results}

Simulation results showing characteristics of voltage dips caused by a short-circuit fault, transformer energizing and motor starting are shown in Fig. 4(a) through Fig. 8(a). As can be seen in Fig. 4 (a) and Fig. 5(a), voltage dips caused by short-circuit faults have typical characteristics- such events consist of at least two-transition (first-transition and second-transition) segments, and a during-event segment. Whereas, voltage events caused by transformer energizing (Fig. 6(a) and Fig. 7(a)) and motor starting (Fig. 8(a)) have

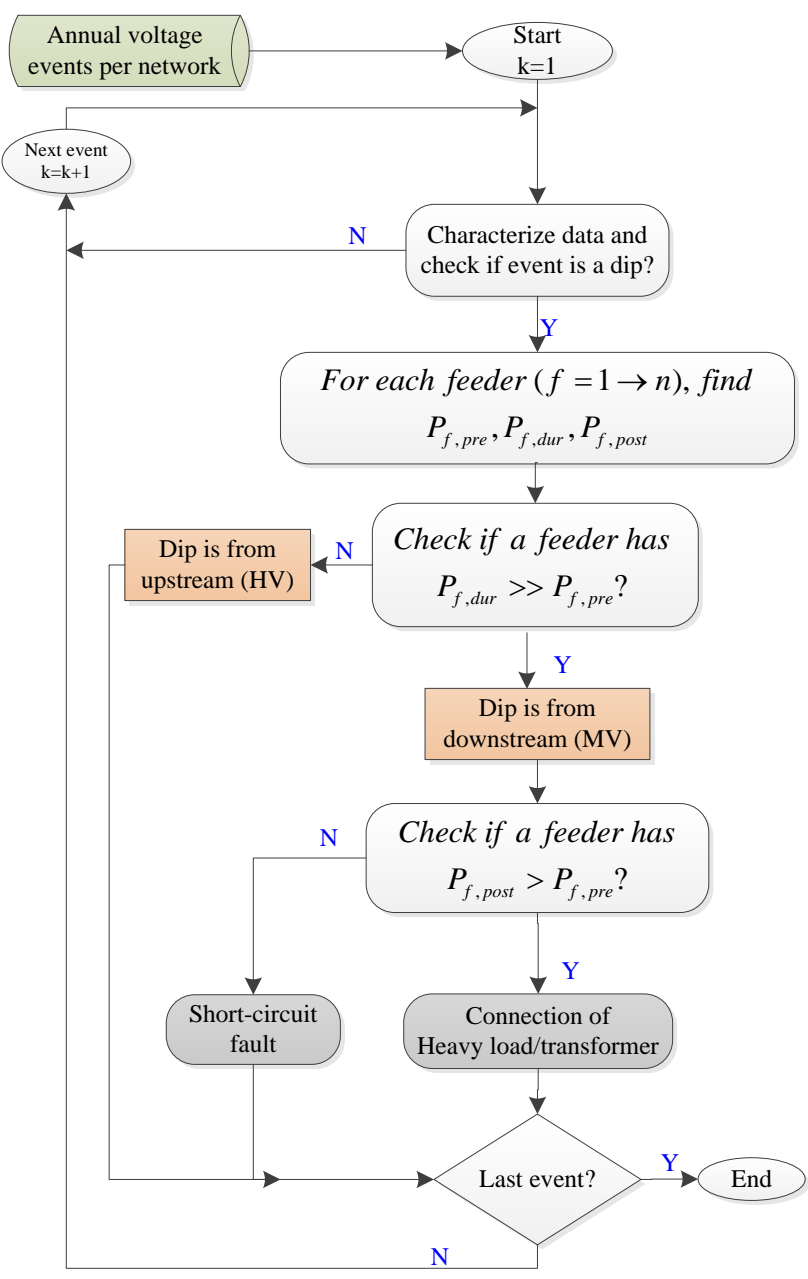

Fig. 3. Flow-chart for detecting the source of voltage dips using active power method

one-transition segment- when slowly recovering from the minimal to about nominal value; and it can also be noticed that such voltage events do not include clear during-event segment. Voltage disturbances caused by transformer energizing have unbalanced voltages while that of motor starting show balanced voltages with longer recovery time. The shape of voltages could give indications about the cause of voltage dips.

Simulation results for detecting the cause and origin of dips using the proposed method in (b-c) of Fig. 4 through Fig. 8. During short-circuit events in the MV-network (Fig. 4(b-c), the during-dip power in one of the feeders is very big compared to the pre-dip power, and the post-dip power of at least one feeder is smaller than the pre-dip power. When the fault is in the upstream (HV-network) (Fig. 5(b-c)), during-dip powers of downstream feeders are lower than the pre-dip power, and the post-dip powers of the feeders can be reduced or remain the same as compared to the pre-dip powers depending on the severity of the dip and its impact on the loads. This leads to a general concept given by (1) and (2).

$$
\begin{gathered}
\text { Downstream : } \\
\left(P_{f, \text { dur }}>>P_{f, \text { pre }}\right) \cap\left(P_{f, \text { post }} \leq P_{f, \text { pre }}\right) \\
\text { (at least for a feeder })
\end{gathered}
$$



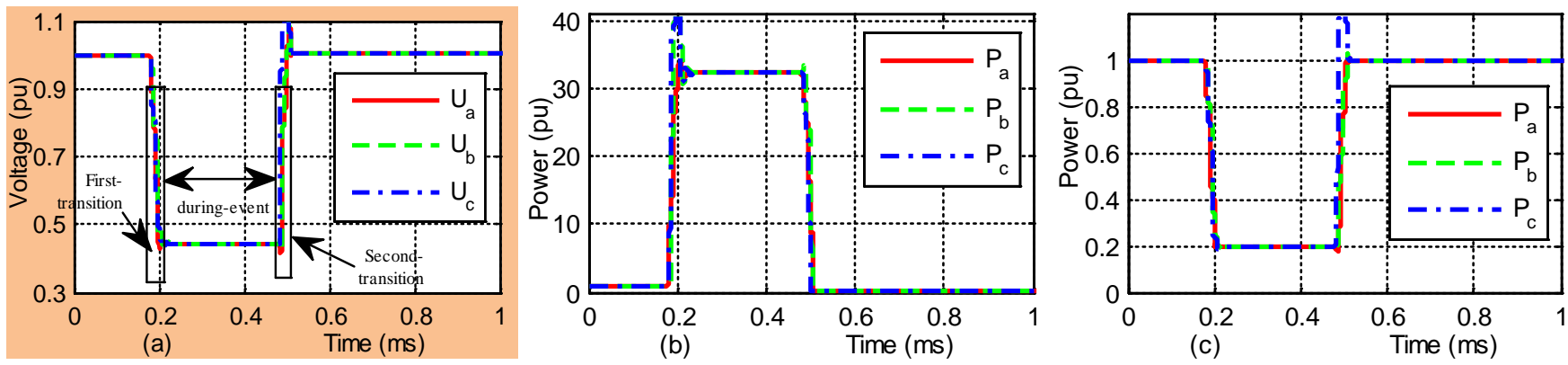

Fig. 4. Short-circuit fault in the MV-network- (a) $U_{a b c}$ at the PCC, (b) Power through feeder-1, (c) power through feeder-2

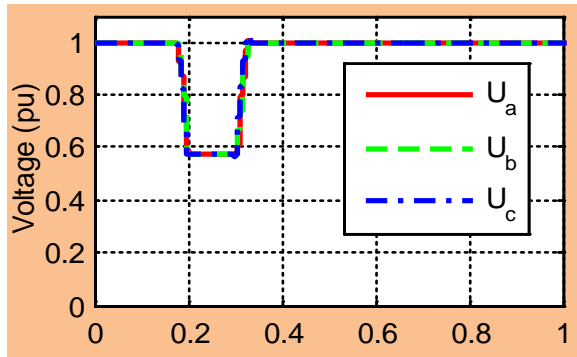

(a) Time (ms)

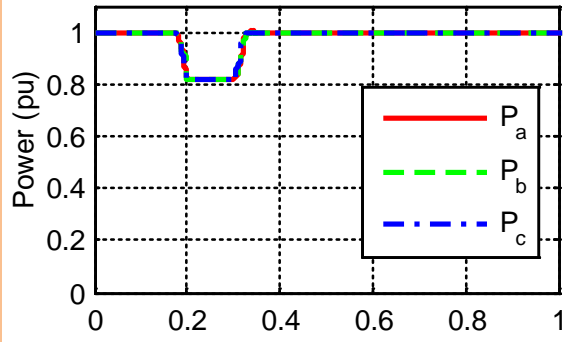

(b)

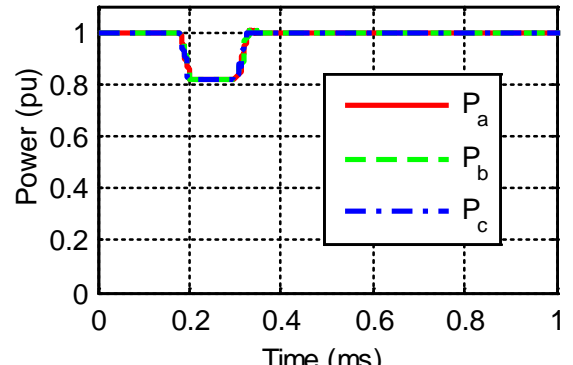

Fig. 5. Short-circuit fault in the HV-network- (a) $U_{a b c}$ at the PCC, (b) Power through feeder-1, (c) power through feeder-2

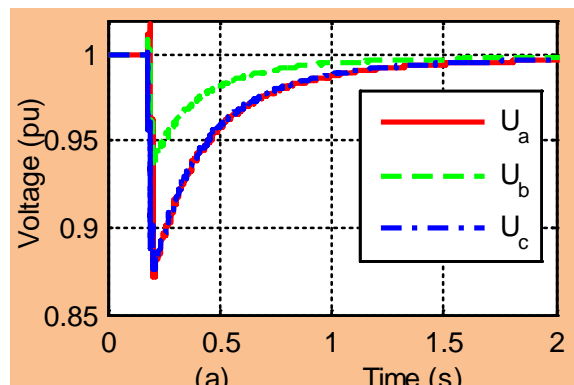

(a)

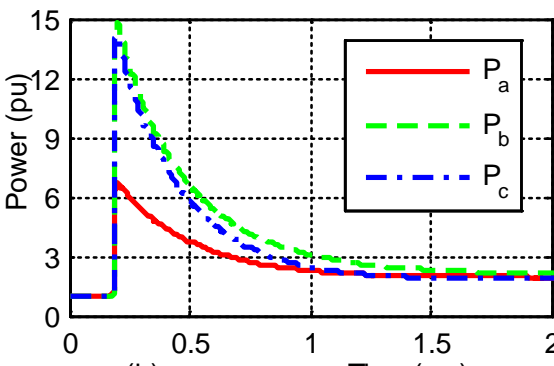

(b)

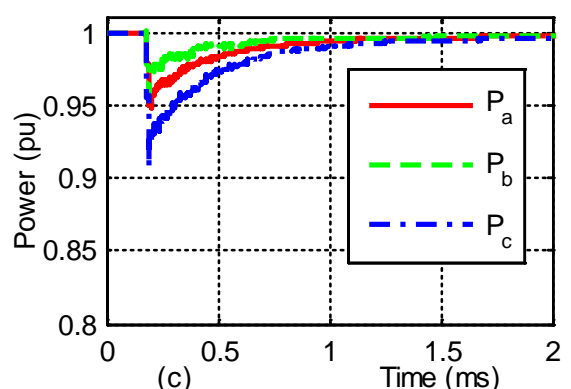

(c)

Fig. 6. Energizing of a transformer in the MV-network- (a) $U_{a b c}$ at the PCC, (b) Power through feeder-1, (c) power through feeder-2
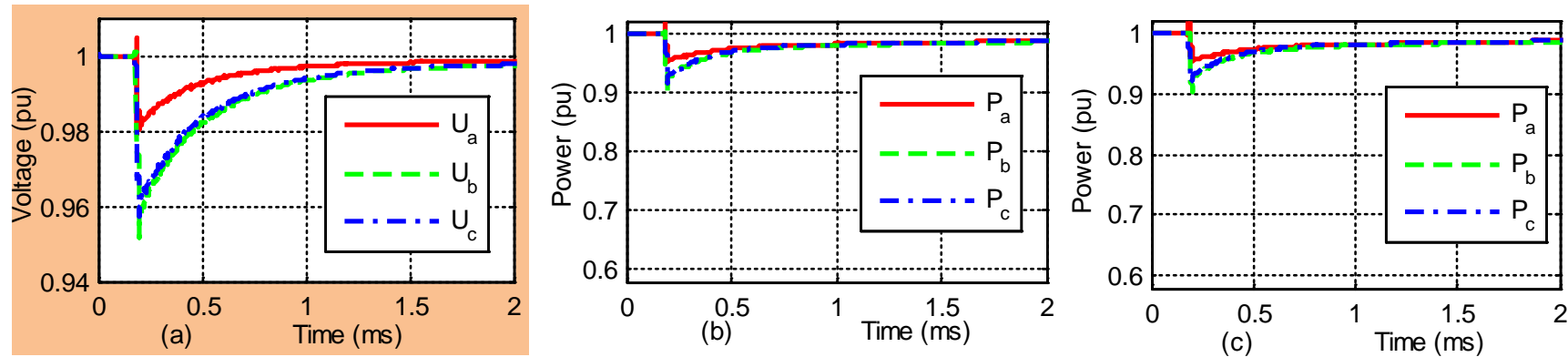

Fig. 7. Energizing of a transformer in the HV-network- (a) $U_{a b c}$ at the PCC, (b) Power through feeder-1, (c) power through feeder- 2
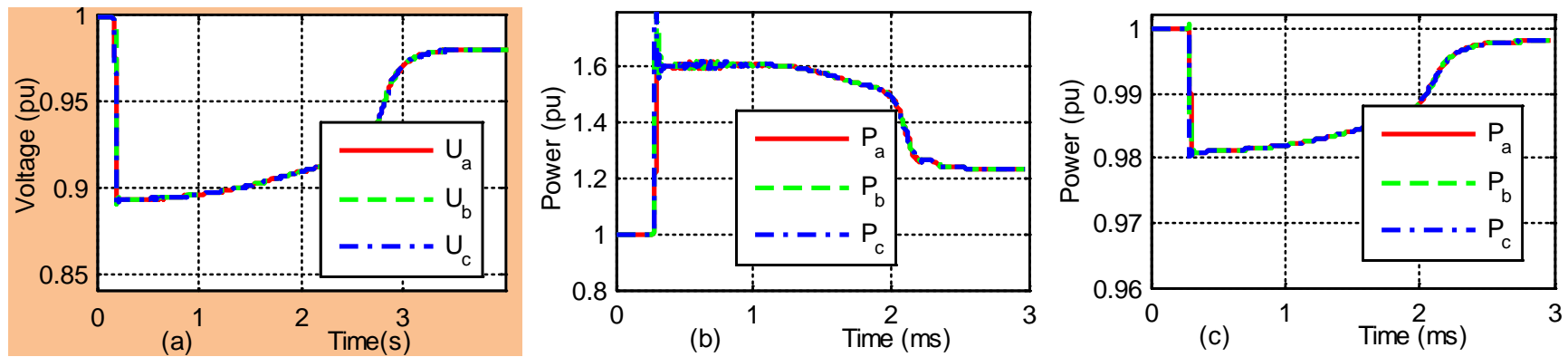

Fig. 8. Connection of a motor in the MV-network- (a) $U_{a b c}$ at the PCC, (b) Power through feeder-1, (c) power through feeder-2

Upstream : $\left(P_{f, \text { dur }} \leq P_{f, \text { pre }}\right) \cap\left(P_{f, \text { post }} \leq P_{f, \text { pre }}\right)$

(for all feeders)
With the connection of a transformer in the MV-network (Fig. 6(b-c)), the feeder where the transformer is 
connected shows very high power while powers of other feeders slightly reduce during the dip event. Besides, the post-dip power of one feeder, where the transformer is connected, increases while that of other feeders are hardly affected compared to the pre-dip powers. When a transformer is connected in the HV-network, the duringdip power of any feeder connected to the PCC may slightly reduce and recovers when the transformer is excited fully (Fig. 7 (b-c)). Generally, the concepts during such situations can be summarized by (3) and (4).

$$
\begin{aligned}
\text { Downstream : } & \left(P_{f, \text { dur }}>>P_{f, \text { pre }}\right) \cap\left(P_{f, \text { post }}>P_{f, \text { pre }}\right) \\
& \text { (at least for a feeder })
\end{aligned}
$$

$$
\begin{gathered}
\text { Upstream : }\left(P_{f, \text { dur }} \leq P_{f, \text { pre }}\right) \cap\left(P_{f, \text { post }} \leq P_{f, \text { pre }}\right) \\
\text { ( for all feeders })
\end{gathered}
$$

Fig. 8 (b-c) shows that the ratio of during-dip and post-dip powers to the pre-dip power during the starting of a motor in a feeder of the MV-network. The feeder where a motor start happens shows relatively higher power during the dip event than before the dip event and its post-dip power also increased because of the motor load, whereas the ratios of post-dip to pre-dip powers are hardly affected in the other feeders. The starting of motors can lead us to the general conclusions given by (5) and (6).

$$
\begin{gathered}
\text { Downstream : }\left(P_{f, \text { dur }}>>P_{f, \text { pre }}\right) \cap\left(P_{f, \text { post }}>P_{f, \text { pre }}\right) \\
(\text { at least for a feeder }) \\
\text { Upstream : }\left(P_{f, \text { dur }} \leq P_{f, \text { pre }}\right) \cap\left(P_{f, \text { post }} \leq P_{f, \text { pre }}\right) \\
(\text { for all feeders })
\end{gathered}
$$

\section{B. Method Applied to Field Data}

In the Netherlands, circuit breakers with fault clearing time up to $100 \mathrm{~ms}$ are commonly used in the HV-networks while circuit breakers having clearing time longer than $100 \mathrm{~ms}$ are used in the MV-networks. A quick but not effective indication into estimating the origin of a dip is by looking at its duration. The proposed method is applied to a dataset of field measurements from a $10 \mathrm{kV}$ MV distribution network and results are compared with the prediction of dips origin based on their duration.

For a particular network where 22 dips are monitored in four years, results of the proposed method (Method-2) as compared to predicting origin of dips based on duration (Method-1) are shown in Fig. 9. As can be seen in Fig. 9, the origin of majority ( $73 \%$ ) of the dips match in both

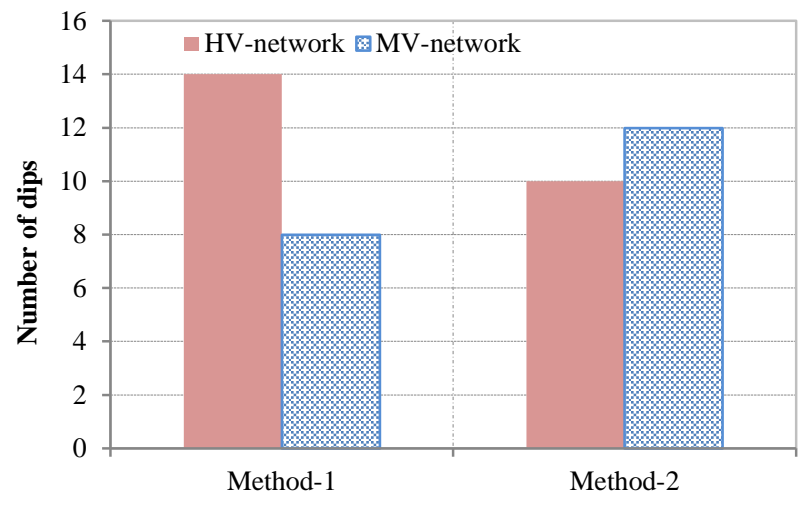

Fig. 9. Origin of dips estimated by method-1 and method-2

methods while the origin of 6 dips, as summarized in TABLE II, are different in both methods and the source of the errors is from Method- 1 in that:

- 5 events estimated as if they originate from the HVnetwork by Method-1 (based on dip-duration) are actually originating from the MV-network caused by energizing of transformers (4) and staring of heavy load (1). As the duration of those dips are short, Method-1 considered them as if they are from the HV-network while Method-2 detected them correctly.

- 1 event that has very long duration is predicted by Method-1 as if it originates from the MV-network. However, the event is caused by short-circuit fault in the HV-network resulting in a multiple-dips. The duration is very long because of the aggregation method applied to multi-dip events. In this paper, the duration of such situations is considered as the interval between the instant of the voltage below threshold of the first dip and the instant of the voltage when it crosses the threshold value during recovery of the last dip.

Although dip-duration can help to make quick prediction regarding the origin of voltage dips, it is not reliable and may lead to erroneous conclusions. Aggregation methods with short multi-dip events can lead long dip-durations. Besides, dip-durations obtained from the shapes of voltages during disturbances may not exactly correspond

\begin{tabular}{|c|c|c|c|c|c|c|c|}
\hline Dip & Ures & Duration & Type & Multi-dip & Orig̨ & gin & \\
\hline event & (pu) & (ms) & $(1,2,3)$ & (\#) & Method-1 & Method-2 & Explanation \\
\hline 1 & 0.85 & 10 & \begin{tabular}{r|}
1 \\
\end{tabular} & 1 & $\mathrm{HV}$ & MV & Short-dip event caused by transformer excitation in the MV-network \\
\hline 2 & 0.63 & 2730 & 1 & 3 & MV & HV & Long-dip event that consits of multiple-dips in the HV-network \\
\hline 3 & 0.87 & 50 & 2 & 1 & $\mathrm{HV}$ & MV & Short-dip event caused by transformer excitation in the MV-network \\
\hline 4 & 0.81 & 30 & 2 & 1 & $\mathrm{HV}$ & MV & Short-dip event due to transformer excitation in the MV-network \\
\hline 5 & 0.73 & 70 & 2 & 1 & $\mathrm{HV}$ & MV & Short-dip event caused by transformer excitation in the MV-network \\
\hline 6 & 0.83 & 100 & 3 & 1 & $\mathrm{HV}$ & MV & Short-dip event due to starting of heavy load in the MV-network \\
\hline
\end{tabular}
to the settings of the protection device- for instance, with multi-stage dips. Short-dips may also originate from the MV-networks due to transformer excitations or staring of loads. The approach, proposed in this paper, considers the actual increase/decrease of power as a consequence of voltage events caused by various phenomenon. The

TABLE II. COMPARISON OF METHODS WHEN APPLIED TO FIELD MEASUREMENTS 
limitations of Method-1 in identifying the origin of dips with multi-dip events, developing-dips, or short-duration dips caused by the excitation of transformers or motor starting in the MV-network can be resolved by Method-2. Besides, the impact of voltage dips can be studied and their severity can related be to the origin and cause of dips.

\section{Conclusion}

By analysing the shape and dip-duration of RMS voltages at the PCC, DSOs can get quick estimation about the source of dips using one monitor. This method is simple but it can lead to erroneous conclusion with multi-dip events and with dips caused by energizing of transformers or starting of motors in the MV-network. From data of a single monitor in a MV distribution network, a method based on the active power of feeders is discussed in this paper. The latter is more efficient method than the former as it considers the actual increase/decrease of powers as a consequence of disturbances and their impacts to the aggregated customers of the feeders.

\section{REFERENCES}

[1] "EN 50160(2010- Voltage characteristics of electricity supplied by public electricity networks," ed. Brussels: CENELEC, 2010.

[2] "IEC 61000-2-8- Voltage dips and short interruptions on public electric power supply systems with statistical measurement results," ed. Switzerland: International Electrotechnical Commision (IEC), 2002.

[3] M. H. J. Bollen and L. D. Zhang, "Analysis of Voltage Tolerance of AC Adjustable-Speed Drives for Three-Phase Balanced and Unbalanced Sags," IEEE Transaction on Power Delivery, vol. 36, pp. 904-910, June 2000.

[4] S.Ž. Djokic', K. Stockman, J.V. Milanovic, J.J.M. Desmet, and R. Belmans, "Sensitivity of AC Adjustable Speed Drives to Voltage Sags and Short Interruptions," IEEE Transaction on Power Delivery, vol. 20, pp. 494-505, Jan. 2005.
[5] S.Ž. Djokic', J.V. Milanovic, and D. S. Kirschen, "Sensitivity of AC Coil Contactors to Voltage Sags, Short Interruptions, and Under-voltage Transients," IEEE Transaction on Power Delivery, vol. 19, pp. 1299-1307, July 2004.

[6] S. Ž. Djokic', J. Desmet, G. Vanalme, J. V. Milanovic', and K. Stockman, "Sensitivity of Personal Computers to Voltage Sags and Short Interruptions," IEEE Transaction on Power Delivery, vol. 20, pp. 375-383, Jan. 2005.

[7] J. Manson and R. Targosz, "Leonardo PQ initiative teamEuropean Power Quality Survey Report," Nov. 2008.

[8] L. E. Weldemariam, V. Cuk, J. F. G. Cobben, and W. L. Kling, "Monitoring of Dips in the MV Network for Regulation- A Case Study," in IEEE Proc. of $16^{\text {th }}$ International Conference on Harmonics and Quality of Power (ICHQP), Bucharest, Romania, 25-28 May 2014.

[9] M. Piumetto and J. G. Targarona, "Characterization of Voltage Sags and its Impact on Sensitive Loads in a MV System with Distributed Generation for Single-phase Fault," IEEE Latin America Transaction, vol. 11, pp. 439-446, Feb. 2013.

[10] "Voltage Dip Immunity of Equipment and InstallationsCIGRE/CIRED/UIE Joint Working Group C4.110," Apr. 2010.

[11] G. Jie, L. Qun-zhan, and W. Jia, "Method for Voltage Sag Disturbance Source Location by the Real Current Component " in Proc. of IEEE on Power and Energy Engineering Conference (APPEEC), 2011 Asia-Pacific, Wuhan, Mar. 2011.

[12] C. Li, T. Tayjasanant, W. Xu, and X. Liu, "Method for voltagesag-source detection by investigating slope of the system trajectory," IEE Proc. Gener. Transm. Distrib., vol. 150, pp. $367-$ 372, 2003.

[13] A. K. Pradhan and A. Routray, "Applying distance relay for voltage sag source detection," IEEE Transaction on Power Delivery, vol. 20, Jan. 2005.

[14] S. Ahn, D. Won, C. I, and S. Moon, "A New Approach to Determine the Direction and Cause of Voltage Sag," Journal of Electrical Engineering \& Technology, vol. 3, pp. 300-307, 2008.

[15] R. Chiumeo, L. Garbero, F. Malegori, and L. Tenti, "Feasible Methods to Evaluate Voltage Dips Origin," in Proc. of the $13^{\text {th }}$ International Conference on Renewable Energies and Power Quality, La Coruña, Spain, Mar. 2015.

[16] M. H. J. Bollen, "Voltage Sags in Three-Phase Systems," in IEEE Power Engineering Review, Sep. 2001.

[17] "IEC 61000-4-30: Testing and measurement techniques - Power quality measurement methods," ed. Switzerland: IEC, 2008. 